\title{
ARENS REGULARITY AND RETRACTIONS
}

\author{
by NILGÜN ARIKAN
}

(Received 13 April, 1981)

In this paper a characterisation of the regularity of a normed algebra $A$ is given in terms of retractions onto $A^{* *}$ from $A^{4 *}$. The second dual $A^{* *}$ of a normed algebra $A$ possesses two natural Banach algebra multiplications, say $\circ$ and $*$. Each of $\circ$ and $*$ extends the original algebra multiplication on $A$; see (2). An algebra $A$ is called regular if and only if $F * G=F \circ G$ for all $F, G \in A^{* *}$. See (1). The existing results in the Arens regularity theory can be found in a recent survey (2). Denoting the $n$th dual of $A$ by $A^{n *}$, and $e_{n}$ the natural embedding of $A^{n *}$ in its second dual $A^{(n+2) *}$, we can naturally represent the second dual $A^{* *}$ of $A$ as a Banach space retract of $A^{4 *}$ in two different ways:

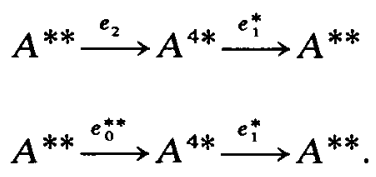

Our main results say that $A^{* *}$ is in fact a Banach algebra retract of $A^{4 *}$ (i.e. the maps involved are homomorphisms) in either of these cases if and only if $A$ is regular.

The notation in (2) for the Arens multiplication is not easy to use for our purposes and we shall first establish our own. We consider the following bilinear mappings:

and

$$
\begin{aligned}
A^{*} \times A & \rightarrow A^{*}:(f, x) \rightarrow{ }_{x} f, & \text { where } & { }_{x} f(y)=f(x y), \\
A^{* *} \times A^{*} & \rightarrow A^{*}:(G, f) \rightarrow f_{G}, & \text { where } & f_{G}(x)=G\left({ }_{x} f\right),
\end{aligned}
$$

$$
A^{* *} \times A^{* *} \rightarrow A^{* *}:(F, G) \rightarrow F \circ G \text {, where } F \circ G(f)=F\left(f_{G}\right) .
$$

Similarly, we consider the following bilinear mappings:

$$
\begin{array}{rlll}
A^{*} \times A & :(f, y) \rightarrow f_{y}, & \text { where } & f_{y}(x)=f(x y), \\
A^{* *} \times A^{*} & :(F, f) \rightarrow{ }_{F} f, & \text { where } & { }_{F} f(y)=F\left(f_{y}\right),
\end{array}
$$

and

$$
A^{* *} \times A^{* *} \rightarrow A^{* *}:(F, G) \rightarrow F * G, \text { where } F * G(f)=G\left({ }_{F} f\right) .
$$

It is easy to see that each of $\circ$ and $*$ is a Banach algebra multiplication on $A^{* *}$ extending the original algebra multiplication on $A$. We call $\circ$ (respectively $*$ ) the first (second) Arens multiplication on $A^{* *}$.

The algebra formed by $A^{* *}$ with its first Arens multiplication will be denoted by $\left(A^{* *}, \circ\right)$. The second dual of $\left(A^{* *}, \circ\right)$ with its first Arens multiplication will be denoted by $\left(A^{4 *}, \infty\right)$, and with its second by $\left(A^{4 *}, \circ *\right)$. In the same way, the algebras $\left(A^{4 *}, * \circ\right)$ and $\left(A^{4 *}, * *\right)$ are second duals of $\left(A^{* *}, *\right)$. Where there is no possibility of confusion, we may write simply oo for the algebra $\left(A^{4 *}, \circ\right)$ etc. 
Because the canonical embedding of an algebra into its second dual is always a homomorphism, the maps

$$
\begin{array}{ll}
e_{2}: \circ \rightarrow \circ & e_{2}: * \rightarrow * \circ \\
e_{2}: \circ \rightarrow \circ * & e_{2}: * \rightarrow * *
\end{array}
$$

are homomorphisms (and in fact, injective homomorphisms). On the other hand,

$$
e_{2}: \circ \rightarrow * \circ
$$

sends $\left(A^{* *}, \circ\right)$ onto $e_{2}\left(A^{* *}\right)$ with the multiplication induced by $* \circ$, i.e. onto a copy of $\left(A^{* *}, *\right)$. From this observation, it is easy to see the result.

PROPOSITION 1. The following are equivalent:

(i) $A$ is regular.

(ii) $e_{2}: \circ \rightarrow * \circ$ is a homomorphism;

(iii) $e_{2}: \circ \rightarrow * *$ is a homomorphism.

Proposition 2. Let $A$ be a regular normed algebra. Then $A^{* *}$ is a Banach algebra retract of $A^{4 *}$.

Proof. Under the regularity hypothesis on $A$ there is just one multiplication ${ }^{\circ}$ on $A^{* *}$. We shall prove that $e_{1}^{*}:\left(A^{4 *}, \circ \circ\right) \rightarrow\left(A^{* *}, \circ\right)$ is a homomorphism (the proof for the other multiplication, ${ }^{*}$, on $A^{4 *}$ is similar). First, for $f \in A^{*}, F \in A^{* *}$ we prove that ${ }_{F} e_{1}(f)=e_{1}\left({ }_{F} f\right)$. Indeed, for each $G \in A^{* *}$ we find

$$
\left({ }_{F} e_{1}(f)\right)(G)=e_{1}(f)(F \circ G)=F \circ G(f)=G\left({ }_{F} f\right)=e_{1}\left({ }_{F} f\right)(G)
$$

(where the regularity of $A$ has been used). Next, if $\Psi \in A^{4 *}$, we prove that $e_{1}(f)_{\Psi}=$ $e_{1}\left(f_{e_{i}^{*}(\Psi)}\right)$; indeed, for each $F \in A^{* *}$,

$$
\begin{aligned}
\left(e_{1}(f)_{\Psi}\right)(F) & =\Psi\left({ }_{F} e_{1}(f)\right)=\Psi\left(e_{1}\left({ }_{F} f\right)\right) \\
& =e_{1}^{*}(\Psi)\left({ }_{F} f\right)=F\left(f_{e_{1}^{*}(\Psi)}\right) \\
& =e_{1}\left(f_{e_{1}^{*}(\Psi)}\right)(F) .
\end{aligned}
$$

Finally, if also $\Phi \in A^{4 *}$, we have

$$
\begin{aligned}
e_{1}^{*}(\Phi \circ \circ \Psi)(f) & =\Phi \circ \% \Psi\left(e_{1}(f)\right)=\Phi\left(e_{1}(f)_{\Psi}\right) \\
& =\Phi\left(e_{1}\left(f_{e_{1}^{*}(\Psi)}^{*}\right)\right)=e_{1}^{*}(\Phi)\left(f_{e_{1}^{*}(\Psi)}\right) \\
& =e_{1}^{*}(\Phi) \circ e_{1}^{*}(\Psi)(f) .
\end{aligned}
$$

Lemma 3. Let $X, Y, Z, W$ be topological spaces with $X$ compact and $Y$ compact Hausdorff. Also assume that there exist mappings $\Phi, \Psi, \chi$ and $\omega$ with $\Phi, \Psi, \chi$ continuous and $\Phi$ surjective such that the following diagram commutes.

Then $\omega$ is continuous.

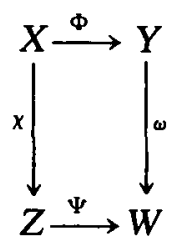


Proof. The proof follows easily from the identity $\omega^{-1}(K)=\Phi\left(\chi^{-1}\left(\Psi^{-1}(K)\right)\right)(K \subseteq W)$.

THEOREM 4. Let $A$ be a normed algebra and $A^{*}, A^{* *}, A^{3 *}$ and $A^{4 *}$ denote its first, second, third and fourth duals respectively. Also let $e_{1}: A^{*} \rightarrow A^{3 *}$ represent the canonical embedding of $A^{*}$ into its second dual $A^{3 *}$. In order that $A$ be regular it is necessary and sufficient that $e_{1}^{*}$ be a homomorphism from $A^{4 *}$ with any one of its four multiplications to $A^{* *}$ with either of its two multiplications.

Proof. Necessity is given in the course of Proposition 2. Sufficiency will be proved here in the case when $e_{1}^{*}: 00 \rightarrow \circ$ is a homomorphism (the other cases are proved in the same way).

Let $F$ in $A^{* *}$ be fixed. The map $e_{1}^{*}: A^{4 *} \rightarrow A^{* *}$ is surjective as is also the map $e_{1}^{*}: A_{1}^{4 *} \rightarrow A_{1}^{* *}$ (where the suffix 1 on a space denotes its unit ball), and these two maps are continuous when their domains and ranges are given their weak* topologies. Now consider the following diagram

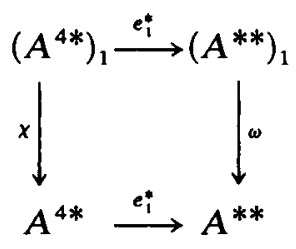

with $\chi: \Psi \rightarrow e_{2}(F) \circ \Psi$ and $\omega: G \rightarrow F \circ G$. It is easy to prove that the diagram commutes when $e_{1}^{*}: \circ \circ \rightarrow \circ$ is a homomorphism. The weak* continuity of $\chi$ follows from the fact that for any Banach algebra $B$, the mapping $x \mapsto y \circ x$ in $B^{* *}$ is weak* continuous provided that $y \in e_{0}(B)$ where $e_{0}: B \rightarrow B^{* *}$ is the natural embedding; see $[2, \mathrm{p}$. 311]. By applying Lemma 3 we deduce that $\omega$ is weak* continuous on the right on $\left(A^{* *}\right)_{1}$. Now the regularity of $A$ follows since the first Arens product is seen to be separately weak* continuous; see Theorem 1 of (2).

COROLlary 5. Let $A$ be a normed algebra with $A^{n *}$ denoting its $n$ th-dual space, and $e_{n}: A^{n *} \rightarrow A^{(n+2) *}$ be the natural embedding of $A^{n *}$ into its second dual $A^{(n+2) *}$. In order that all $2^{k}$ possible Arens multiplications on $A^{2 k *}$ coincide it is necessary and sufficient that $e_{2 k-1}^{*}$ be a homomorphism for any Arens products on $A^{(2 k+2) *}$ and $A^{2 k *}$.

Proof. For $k=1$, the corollary is Theorem 4. Assume it holds for $k=r$. The diagram

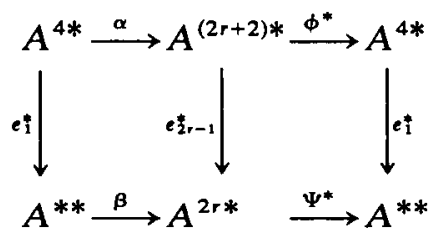

commutes for $\alpha, \beta$ the natural inclusions and $\phi^{*}, \Psi^{*}$ the adjoints of the natural inclusions $\phi: A^{3 *} \rightarrow A^{(2 r+1) *}$ and $\Psi: A^{*} \rightarrow A^{(2 r-1) *}$ showing that the restriction of $e_{2 r-1}^{*}$ to $A^{4 *}$ is just $e_{1}^{*}$. Thus, when $A^{4 *}$ (respectively $A^{* *}$ ) has the Arens multiplication induced from 
$A^{(2 r+2) *}$ (respectively $\left.A^{2 r *}\right), e_{1}^{*}$ is a homomorphism. By Theorem $4, A$ is regular. Put $B=A^{* *}$ with its (unique) multiplication. Then $B^{(2 k-2) *}=A^{2 k *}$ etc., so we may apply the inductive hypothesis to conclude that all Arens multiplications on $B^{* *}=A^{4 *}$ coincide and so on.

Conversely, assume that all $2^{k}$ possible Arens multiplications on $A^{2 k *}$ coincide. Then by Theorem 4 it follows that $e_{2 k-1}^{*}$ is a homomorphism.

Corollary 5 allows us to speak of the regularity of $A^{(2 k-1) *}$ without specifying the multiplication on it (as all are identical). N. J. Young produced an example in (3) to show that $A$ could be regular although $A^{* *}$ was not. We do not yet know examples in which $A^{2(k-1) *}$ is regular but $A^{2 k *}$ is not for $k>1$.

So far we have been considering the natural inclusion $e_{2}$ of $A^{* *}$ into $A^{4 *}$, but there is another natural map from $A^{* *}$ to $A^{4 *}$, namely the second adjoint $e_{0}^{* *}$ of the natural inclusion $e_{0}: A \rightarrow A^{* *}$. The mappings $e_{0}^{* *}$ and $e_{2}$ are very different, as the following Proposition shows.

Proposition 6. For a Banach algebra $A, e_{0}^{* *}\left(A^{* *}\right) \cap e_{2}\left(A^{* *}\right)=e_{2}\left(e_{0}(A)\right)$ where $e_{0}, e_{2}, e_{0}^{* *}$ are defined as above.

Proof. It is clear that the right hand side is contained in the left hand side. So let $\Phi \in e_{0}^{* *}\left(A^{* *}\right) \cap e_{2}\left(A^{* *}\right) \backslash e_{2}\left(e_{0}(A)\right)$. There exists $F \in A^{* *}$ and $G \in A^{* *} \backslash e_{0}(A)$ for which $\Phi=e_{0}^{* *}(F)=e_{2}(G)$. As $A$ is weak ${ }^{*}$ dense in $A^{* *}$ there is a net $\left(x_{i}\right)$ in $A$ with $F=$ $w^{*} \lim _{i} e_{0}\left(x_{i}\right)$. So for each $\sigma \in A^{3 *}$ we have

$$
\begin{aligned}
\Phi(\sigma) & =e_{0}^{* *}(F)(\sigma)=\lim _{i} e_{0}^{* *}\left(e_{0}\left(x_{i}\right)\right)(\sigma) \\
& =\lim _{i} \sigma\left(e_{0}\left(x_{i}\right)\right)
\end{aligned}
$$

and $\Phi(\sigma)=e_{2}(G)(\sigma)=\sigma(G)$. By choosing a $\sigma \in A^{3 *}$ with $\sigma\left(e_{0}(A)\right)=0$ and $\sigma(G)=1$ we obtain a contradiction. Thus the proof is completed.

Now $e_{0}: A \rightarrow A^{* *}$ is a homomorphism when $A^{* *}$ has either of its Arens multiplications; because the second adjoint of a homomorphism is a homomorphism provided that the same Arens multiplication is taken in each case, we have that

$$
\begin{array}{ll}
e_{0}^{* *}: \circ \rightarrow \circ & e_{0}^{* *}: * \rightarrow \circ * \\
e_{0}^{* *}: \circ \rightarrow * \circ & e_{0}^{* *}: * \rightarrow * *
\end{array}
$$

are homomorphisms. Obviously, if $\circ=*$, then

$$
e_{0}^{* *}: \circ \rightarrow \circ * \quad e_{0}^{* *}: \circ \rightarrow * *
$$

are also homomorphisms. The converse is also true.

Proposition 7. Let $A$ be a normed algebra and $e_{0}: A \rightarrow A^{* *}$ be its canonical embedding. Then $A$ is regular if and only if either of the following is an algebra homomorphism:

$$
e_{0}^{* *}: \circ \rightarrow \circ * \quad e_{0}^{* *}: \circ \rightarrow * * .
$$

Proof. We will only give the proof that $A$ is regular when $e_{0}^{* *}: \circ \rightarrow * *$ is a 
homomorphism. So for $F, G \in A^{* *}$ and $\sigma \in A^{3 *}$ we have

$$
e_{0}^{* *}(F \circ G)(\sigma)=e_{0}^{* *}(F) * * e_{0}^{* *}(G)(\sigma) .
$$

But the right hand side of the latter equality is $e_{0}^{* *}(F * G)(\sigma)$ since $e_{0}^{* *}: * \rightarrow * *$ always is a homomorphism. Thus

$$
F \circ G\left(e_{0}^{*}(\sigma)\right)=F * G\left(e_{0}^{*}(\sigma)\right),
$$

and the proof is completed by recalling that $e_{0}^{*}: A^{3 *} \rightarrow A^{*}$ is a surjection.

COROLlaRY 8. The mappings

$$
A^{* *} \stackrel{e_{0}^{* *}}{\longrightarrow} A^{4 *} \stackrel{e_{i}^{*}}{\longrightarrow} A^{* *} .
$$

present $A^{* *}$ as a retraction of $A^{4 *}$ (for any of the Arens multiplications on $A^{* *}$ and $A^{4 *}$ ) if and only if $A$ is regular

Proof. This is clear.

I am grateful to Professor J. S. Pym for his advice and interest on this work.

\section{REFERENCES}

1. F. F. Bowall and J. Duncan, Complete normed algebras (Springer-Verlag, 1973).

2. J. Duncan and S. A. R. Hosseinian, The second dual of a Banach algebra. Proc. Roy. Soc. Edinburgh 84(A), (1979), 309-325.

3. N. J. Young, Periodicity of functionals and representations of normed algebras on reflexive spaces. Proc. Edinburgh Math. Soc., (2) 20, (1976), 99-120.

Department of Pure Mathematics

UNIVERSITY OF SHEFFIELD

SHEFFIELD S10 2TN
Present address:

DePARTMENT OF MATHEMATiCS BOSPHORUS UNIVERSITY Bebex, Istanbul 